\section{Insuficiência renal aguda em pacientes internados por insuficiência cardíaca descompensada - Reincade}

\author{
Acute kidney injury in hospitalized patients with decompensated \\ heart failure
}

\begin{abstract}
Autores
Larissa Cristina Nascimento de Barros Fábio Serra Silveira Marcos Serra Silveira Thamara Carvalho Morais Marco Antônio Prado Nunes Kleyton de Andrade Bastos
\end{abstract}

Universidade Federal do Sergipe - UFS.

Data de submissão: 17/08/2011 Data de aprovação: 04/01/2012

\section{Correspondência para: Larissa Cristina Nascimento de Barros Av. Hermes Fontes, 2022, Cond. Morada das Árvores Edifício Jacarandá, ap. 1104 Bairro Grageru Aracaju - SE - Brasil CEP 49020-026 \\ E-mail: larissa_se@} hotmail.com

O referido estudo foi realizado na Fundação de Beneficência Hospital de Cirurgia - FBHC.

Os autores declaram a inexistência de conflitos de interesse.

\section{Resumo}

Introdução: Insuficiência renal aguda (IRA) acontece com frequência em pacientes críticos, porém a significância clínica de sua ocorrência não tem sido determinada na insuficiência cardíaca descompensada (ICD). Objetivos: Estudar a ocorrência e valor prognóstico da IRA em pacientes internados por ICD e avaliar comparativamente com aqueles sem a complicação o perfil clínico-laboratorial e a mortalidade intra-hospitalar. Métodos: Estudo prospectivo em 85 pacientes internados em terapia intensiva (UTI) por ICD entre março de 2010 e fevereiro de 2011. Foi feito o diagnóstico de insuficiência cardíaca (IC) conforme critérios de Boston (escala $\geq 8$ ) e exames complementares, e o diagnóstico de IRA utilizando a classificação AKIN. Na análise estatística, utilizaram-se teste $t$ de Student, qui-quadrado e modelo de regressão com múltiplas variáveis, considerando estatisticamente significativo $\mathrm{p}<0,05$. Resultados: Predominaram homens (55\%), valvopatia foi a principal etiologia da IC (42,4\%), e má aderência/medicação inadequada foi a principal causa de descompensação $(22,4 \%)$. IRA ocorreu em $76,5 \%$ dos indivíduos (4,7\% estágio 1, 32,9\% estágio 2 e $38,8 \%$ estágio 3 AKIN). Havia mais pacientes com anemia $(p=0,01)$ e acima de 60 anos $(\mathrm{p}=0,02)$ no grupo com IRA quando comparado ao controle. Todos os pacientes com disfunção renal prévia desenvolveram IRA. Em média, permaneceram internados por 7,7 dias (grupo IRA $8,8 \pm 6,6$ dias; grupo não IRA $4 \pm 1,4$ dias; $\mathrm{p}<0,01)$. A mortalidade foi maior no grupo com IRA ( $p=0,04)$, principalmente no estágio $3(\mathrm{p}<0,01)$. Identificou-se como preditor independente de IRA o número de dias em UTI $(p=0,02)$. IRA foi o

\section{Abstract}

Introduction: Acute kidney injury (AKI) occurs frequently in critical patients, but its clinical relevance has not been determined in decompensated heart failure (DHF). Objectives: To study the occurrence and prognostic value of $\mathrm{AKI}$ in patients with DHF and to compare the clinical and laboratory characteristics and in-hospital mortality with those without AKI. Methods: Prospective study of 85 patients hospitalized in intensive care unit (ICU) with DHF from March 2010 to February 2011. Diagnosis of heart failure (HF) was established using the Boston criteria (scale $\geq 8$ ) and additional tests, and AKI was defined using the AKIN classification. Patients data with and without AKI were compared using Student's $t$-test, chi-squared statistic and multiple logistic regression, considering statistically significant $\mathrm{p}<0.05$. Results: Most patients were male $(55 \%)$, valvular disease was the main etiology of HF $(42.4 \%)$, and inadequate medication was the main cause of decompensation $(22.4 \%)$. AKI occurred in $76.5 \%$ of patients $(4.7 \%$ stage $1,32.9 \%$ stage 2 and $38.8 \%$ stage 3$)$. Patients were more anemic $(\mathrm{p}=0.01)$ and had over 60 years $(p=0.02)$ in the AKI-group when compared to control. All patients with chronic kidney failure developed AKI. The duration of ICU stay was longer for the AKI group (group AKI $8.8 \pm 6.6$ days; group non-AKI $4 \pm 1.4$ days, $\mathrm{p}<0.01$ ) In-hospital mortality rate was higher in patients with AKI $(\mathrm{p}=0.04)$, especially in stage $3(\mathrm{p}<0.01)$. The duration of ICU stay was an independent predictor of AKI ( $\mathrm{p}=0.02)$. Only AKI was considered as independent predictor of mortality in this group $(p=0,05)$. Conclusion: AKI 
único preditor independente de mortalidade no grupo $(p=0,05)$. Conclusão: IRA ocorre com frequência em ICD, principalmente em estágios AKIN mais avançados, na população renal crônica e idosa, e relacionase a maior tempo de internação e mortalidade.

Palavras-chave: Lesão renal aguda. Insuficiência Cardíaca. Unidades de Terapia Intensiva. is frequent in DHF, especially in advanced stages, in the elderly and patients with chronic kidney disease, and was associated with longer hospitalization and higher mortality rate.

Keywords: Acute Kidney Injury. Heart failure. Intensive Care Units.

\section{INTRODUÇÃO}

A elevada prevalência de insuficiência cardíaca (IC) na população justifica seu status de problema de saúde pública. Estima-se que nos Estados Unidos a insuficiência cardíaca descompensada (ICD) seja responsável por meio milhão de internações por ano. ${ }^{1}$ De acordo com o DATASUS, a ICD representou a segunda principal causa de hospitalização em 2007 (2,6\% do total), ano no qual foram registradas 293.473 internações, com mortalidade hospitalar de $6 \%{ }^{2}$

Pacientes com ICD grave requerem suporte em unidades de terapia intensiva (UTI), ${ }^{3}$ locais onde a insuficiência renal aguda (IRA), embora subdiagnosticada, tem ocorrência geral descrita de 1 a $30 \%$ a depender do critério utilizado, com mortalidade que varia de 28 a $90 \% .{ }^{4}$

A baixa disponibilidade de biomarcadores específicos dificulta a identificação de IRA. Elevação da creatinina sérica, apesar de ser a principal ferramenta no cotidiano médico, não é considerada marcador de qualidade, posto que sua ampla flutuação e elevação tardia podem gerar hipóteses diagnósticas errôneas. A publicação do critério RIFLE, posteriormente modificado pela classificação AKIN, forneceu importante subsídio científico para a detecção da doença. ${ }^{5,6}$ Segundo essa classificação, divide-se a IRA, a partir da variação basal dos níveis de creatinina sérica e do volume urinário, em três estágios progressivos: risco, injúria e lesão renal. ${ }^{6}$ Vários trabalhos atestaram a aplicabilidade de ambas as classificações, que são aceitas atualmente como principais critérios diagnósticos. ${ }^{4,7-9}$

Apesar de patologias frequentes em pacientes críticos, pouco se conhece sobre a interação entre IRA e ICD..$^{10,11}$ Em casuística publicada recentemente, IRA relacionou-se à internação prolongada e maior mortalidade intra-hospitalar em ICD. ${ }^{12}$

$\mathrm{O}$ presente trabalho visou observar a ocorrência de IRA em pacientes críticos internados por ICD, determinar fatores de risco para sua ocorrência, além de analisar o perfil clínico-demográfico dos indivíduos, tempo de internação em UTI e desfecho final.

\section{Métodos}

Estudo de coorte prospectivo com 106 pacientes portadores de ICD admitidos na Unidade Cardiotorácica da Fundação de Beneficência Hospital de Cirurgia (FBHC), em Aracaju - Sergipe, entre fevereiro de 2010 e março de 2011. O intervalo considerado para a observação foi o compreendido entre a admissão na UTI e a saída desta por alta ou óbito. Estabeleceu-se o diagnóstico de IC conforme os critérios de Boston ${ }^{13}$ com escala maior ou igual a oito (Tabela 1), o qual foi confirmado por meio de exames adicionais (ecocardiografia e radiografia de tórax).

Os dados clínicos foram obtidos por meio do protocolo de pesquisa do Registro Sergipano de Insuficiência Cardíaca Descompensada (REINCADE), do qual o presente trabalho foi uma vertente, e da análise dos prontuários. A coleta de

\section{Tabela 1 CRITÉRIOS DE BOSTON PARA O DIAGNÓSTICO DE INSUFICIÊNCIA CARDÍACA}

\begin{tabular}{lc} 
Categoria I: História & Número de pontos \\
\hline Dispneia de repouso & 4 \\
Ortopneia & 4 \\
Dispneia paroxística noturna & 3 \\
Dispneia ao andar no plano & 2 \\
Dispneia ao subir escada & 1 \\
\hline Categoria II: Exame físico & Número de pontos \\
\hline Taquicardia $91-110$ bpm & 1 \\
\multicolumn{1}{c}{$>110$ bpm } & 2 \\
Elevação da pressão venosa & 2 \\
Elevação da pressão venosa com & 3 \\
edema de membros inferiores & \\
Crepitação pulmonar em bases & 1 \\
Acima das bases & 2 \\
Terceira bulha & 3 \\
Sibilos & 3 \\
\hline Categoria III: Radiografia de tórax & Número de pontos \\
\hline Edema alveolar & 4 \\
Edema intersticial & 3 \\
Derrame pleural bilateral & 3 \\
\hline Adeara &
\end{tabular}

Adaptado de Carlson et al. ${ }^{13}$. 
dados iniciou-se após a autorização do paciente mediante assinatura do termo de consentimento livre e esclarecido. Esse projeto está de acordo com os princípios defendidos na declaração de Helsinki e foi aprovado pelo Comitê de Ética em Pesquisa da Universidade Federal de Sergipe com o número de protocolo 1186.0.000.107-08.

Dos pacientes elegíveis para a pesquisa, obtiveram-se informações imediatamente após a admissão na UTI sobre idade, raça, sexo, comorbidades, fatores de risco e hábitos, etiologia da IC, exames laboratoriais mais recentes (creatinina), causa da descompensação clínica, classe funcional de acordo com a New York Heart Association (NYHA) e peso corporal. Aferiram-se a hemoglobina e creatinina sérica no momento da admissão e 48 horas após e, a partir daí, diariamente. Informações sobre o débito urinário foram coletadas de 6 em 6 horas. Dos 101 pacientes internados com ICD no período, 16 foram excluídos da análise em decorrência da ausência de aferição do débito urinário.

Definiu-se o diagnóstico de IRA conforme a classificação AKIN (Tabela 2) e dividiram-se os pacientes em dois grupos conforme o desenvolvimento de IRA. Indivíduos que apresentaram IRA foram discriminados de acordo com o parâmetro determinante para o diagnóstico, se aumento da creatinina ou redução do débito urinário. $\mathrm{Na}$ eventualidade de ambos terem sido determinantes, priorizou-se aquele temporalmente mais precoce. No tocante ao estadiamento, considerou-se para cada paciente o de maior gravidade atingido no período intra-hospitalar.

\begin{tabular}{|c|c|c|}
\hline Tabela 2 & \multicolumn{2}{|c|}{$\begin{array}{l}\text { CLASSIFICAÇÃO/ESTADIAMENTO ACUTE } \\
\text { KIDNEY INJURY NETWORKING PARA } \\
\text { INSUFICIÊNCIA RENAL AGUDA }\end{array}$} \\
\hline Estágio & Critério creatinina sérica & Critério débito urinário \\
\hline 1 & $\begin{array}{l}\text { Elevação da creatinina } \\
\text { sérica maior ou igual a } \\
0,3 \mathrm{mg} / \mathrm{dL} \text { ou aumento } \\
\text { de } 150 \text { - } 200 \% \text { do valor } \\
\text { basal ( } 1,5 \text { a } 2 \text { vezes) }\end{array}$ & $\begin{array}{l}\text { Menor que } \\
0,5 \mathrm{~mL} / \mathrm{Kg} / \mathrm{h} \text { por } 6 \mathrm{~h}\end{array}$ \\
\hline 2 & $\begin{array}{l}\text { Elevação da creatinina } \\
\text { sérica maior que } 200 \text { a } \\
300 \% \text { do valor basal ( } 2 \\
\text { a } 3 \text { vezes) }\end{array}$ & $\begin{array}{l}\text { Menor que } \\
0,5 \mathrm{~mL} / \mathrm{Kg} / \mathrm{h} \text { por }>12 \mathrm{~h}\end{array}$ \\
\hline 3 & $\begin{array}{l}\text { Elevação da creatinina } \\
\text { sérica maior que } 300 \% \\
\text { do valor basal ( }>3 \\
\text { vezes) ou creatinina } \\
\text { sérica ( } \geq 4,0 \mathrm{mg} / \mathrm{dL} \\
\text { com aumento agudo de } \\
\text { pelo menos } 0,5 \mathrm{mg} / \mathrm{dL} \text { ) }\end{array}$ & $\begin{array}{l}\text { Menor que } \\
0,3 \mathrm{~mL} / \mathrm{Kg} / \mathrm{h} \text { por }>24 \mathrm{~h} \\
\text { ou anúria por } 12 \mathrm{~h}\end{array}$ \\
\hline
\end{tabular}

Adaptado de Mehta et al. ${ }^{6}$.
Realizou-se o diagnóstico de anemia, levando em consideração a concentração de hemoglobina à admissão menor que $11 \mathrm{~g} / \mathrm{dL}$. O paciente foi considerado como portador de doença renal prévia quando o clearance de creatinina calculado a partir do valor de creatinina sérica, obtido previamente à internação, foi menor que $60 \mathrm{~mL} / \mathrm{min}$, sendo o valor considerado aquele da mais recente determinação ambulatorial.

As informações obtidas foram confrontadas e submetidas à análise estatística utilizando o programa SPSS (Statistical Package for Social Sciences) versão 18.0 para Windows. Nos casos de reinternação, o paciente foi considerado como caso novo. A análise descritiva dos dados foi realizada por meio de frequências absolutas e relativas e medidas de tendência central e variabilidade. A análise inferencial foi feita por intermédio do qui-quadrado para as variáveis categóricas, e o teste $t$ de Student para as contínuas, sempre considerando estatisticamente significativo o $\mathrm{p}<0,05$. A fim de explorar mais os efeitos individuais dos preditores envolvidos nos episódios de IRA e na mortalidade, construiu-se modelo de regressão logística, por meio de análise com múltiplas variáveis, incluindo os fatores sociodemográficos e as variáveis que apresentaram $\mathrm{p}<0,2$ na análise não ajustada. $\mathrm{O}$ presente estudo não dispôs de fonte externa de financiamento.

\section{Resultados}

As características clínico-demográficas da amostra encontram-se sumariadas na Tabela 3. Os pacientes desta coorte tiveram média de idade de 53,5 anos $( \pm 16,1)$, e $55 \%$ eram do sexo masculino. A etiologia da IC de maior ocorrência foi a valvar $(42,4 \%)$, seguida de isquêmica $(31,8 \%)$ e idiopática $(11,8 \%)$. Má aderência/medicação inadequada foi a causa de descompensação mais frequente no grupo estudado $(22,4 \%)$.

Dos 85 pacientes incluídos no estudo, observou-se IRA em 65 deles $(76,5 \%)$ durante o tempo de hospitalização na UTI e nestes foi mais frequente o estágio 3 da classificação AKIN (38,8\%). A distribuição dos pacientes segundo a ocorrência ou não de IRA e seu estadiamento conforme a classificação AKIN em função do critério utilizado para o diagnóstico encontrase descrita na Tabela 4.

A Tabela 5 relaciona a incidência de IRA com o perfil clínico-demográfico da amostra estudada. Havia mais indivíduos acima de 60 anos $(\mathrm{p}=0,02)$, com disfunção renal prévia $(\mathrm{p}=0,04)$ e com anemia 
$(\mathrm{p}=0,01)$ no grupo com IRA quando comparados ao grupo sem IRA.

Todos os 12 pacientes com IRC desenvolveram IRA na internação. Destes, apenas 2 pacientes realizaram terapia dialítica, e outro indivíduo com tal indicação faleceu antes de realizar o procedimento. Os outros 9 portadores de doença renal crônica não apresentaram indicação para dialisar.

\begin{tabular}{|c|c|c|}
\hline Tabela 3 & \multicolumn{2}{|c|}{$\begin{array}{l}\text { CARACTERÍSTICAS CLÍNICO-DEMOGRÁFICAS } \\
\text { DOS PACIENTES INTERNADOS POR } \\
\text { INSUFICIÊNCIA CARDÍACA DESCOMPENSADA } \\
(\mathrm{n}=85)\end{array}$} \\
\hline \multicolumn{2}{|c|}{ Características } & Percentual \\
\hline \multicolumn{2}{|c|}{ Idade $<60$ anos } & 57,6 \\
\hline \multicolumn{2}{|c|}{ Sexo masculino } & 55,3 \\
\hline \multicolumn{3}{|c|}{ Cor } \\
\hline \multicolumn{2}{|l|}{ Pardos } & 47,1 \\
\hline \multicolumn{2}{|l|}{ Brancos } & 38,8 \\
\hline \multicolumn{2}{|l|}{ Negros } & 11,8 \\
\hline \multicolumn{3}{|c|}{ Comorbidades } \\
\hline \multicolumn{2}{|c|}{ Hipertensão arterial } & 62,4 \\
\hline \multicolumn{2}{|c|}{ Anemia } & 38,8 \\
\hline \multicolumn{2}{|c|}{ Dislipidemia } & 29,4 \\
\hline \multicolumn{2}{|c|}{ Diabetes mellitus } & 24,7 \\
\hline \multicolumn{2}{|c|}{ Fibrilação atrial } & 14,1 \\
\hline \multicolumn{2}{|c|}{ Nefropatia crônica } & 12,9 \\
\hline \multicolumn{2}{|c|}{$\mathrm{DPOC}^{+}$} & 7,1 \\
\hline \multicolumn{2}{|l|}{$\mathrm{AVE}^{\ddagger}$} & 4,7 \\
\hline \multicolumn{2}{|c|}{ Neoplasia } & 3,5 \\
\hline \multicolumn{3}{|c|}{ Classe funcional da insuficiência cardíaca NYHA } \\
\hline \multicolumn{2}{|c|}{ I } & 8,2 \\
\hline \multicolumn{2}{|l|}{$\|$} & 16,5 \\
\hline \multicolumn{2}{|l|}{ III } & 30,6 \\
\hline \multicolumn{2}{|l|}{ IV } & 44,7 \\
\hline \multicolumn{3}{|c|}{ Etiologia da insuficiência cardíaca } \\
\hline \multicolumn{2}{|c|}{ Valvar } & 42,4 \\
\hline \multicolumn{2}{|c|}{ Isquêmica } & 31,8 \\
\hline \multicolumn{2}{|c|}{ Idiopática } & 11,8 \\
\hline Chagássic & & 4,7 \\
\hline Hipertens & & 3,5 \\
\hline Alcoólica & & 1,2 \\
\hline Restritiva & & 1,2 \\
\hline Causa da de & ompensação & \\
\hline Má aderê & a / Medicação inadequada & 22,4 \\
\hline Evolução & ural & 17,6 \\
\hline Evento isc & mico & 16,5 \\
\hline Infecção & & 16,5 \\
\hline Disfunção & bioprótese valvar & 14,1 \\
\hline Arritmia & & 9,4 \\
\hline Outras ca & & 2,4 \\
\hline Hipertens & arterial não controlada & 1.2 \\
\hline
\end{tabular}

${ }^{\dagger}$ DPOC: Doença pulmonar obstrutiva crônica; ${ }^{\ddagger}$ AVE: Acidente vascular encefálico; ${ }^{\S} \mathrm{NYHA}$ : New York Heart Association.
História de etilismo $(\mathrm{p}=0,05)$ e má aderência/medicação inadequada $(\mathrm{p}=0,07)$ refletiram tendência a relacionar-se com a ocorrência de IRA. Os pacientes que desenvolveram IRA tenderam a ter valores admissionais de hemoglobina mais baixos $(\mathrm{p}=0,07)$. $\mathrm{O}$ percentual de indivíduos com IRA aumentou a cada classe funcional da IC proposta pela NYHA, sem, no entanto, haver relevância quando comparados ao grupo controle $(p=0,72)$. Por meio de análise com múltiplas variáveis, identificou-se que o número de dias de hospitalização em UTI $(p=0,02)$ foi o único preditor independente do desenvolvimento de IRA nesta série (Tabela 6). Não foi incluída presença de disfunção renal prévia na análise multivariada de fatores preditores de IRA, apesar de a evidência dessa relação ser clinicamente muito forte, afinal todos os indivíduos nesta condição desenvolveram a complicação.

O tempo de internação em UTI foi em média 7,7 dias $( \pm 1,8)$ e foi significativamente maior em pacientes que desenvolveram IRA (grupo IRA 8,8 $\pm 6,6$ versus grupo não IRA 4,0 $\pm 1,4$ dias; $\mathrm{p}<0,01)$. A mortalidade intra-hospitalar foi maior no grupo com IRA $(\mathrm{p}=0,04)$, principalmente nos pacientes com maior envolvimento renal, classificados como estágio 3 AKIN $(p<0,01)$. Dezessete pacientes com IRA $(26,1 \%)$ evoluíram a óbito no hospital. Destes, $4(23,5 \%)$ haviam alcançado o estágio 2 AKIN e 13 $(76,5 \%)$ atingiram o estágio 3 . Apenas um paciente sem IRA $(5 \%)$ faleceu. Três pacientes com IRC morreram: o primeiro em decorrência da síndrome coronariana aguda, e os outros dois de sepse. Por meio de análise com múltiplas variáveis (Tabela 7), observouse que IRA $(\mathrm{p}=0,05)$ foi o único fator preditor independente para mortalidade no grupo estudado.

\begin{tabular}{|c|c|c|c|}
\hline Tabela 4 & $\begin{array}{l}\text { DISTRIBL } \\
\text { A OCORR } \\
\text { RENAL A } \\
\text { CONFORI } \\
\text { FUNÇÃO } \\
\text { SEU DIAC }\end{array}$ & $\begin{array}{l}\text { ÃO DOS PACIEI } \\
\text { CCIA OU NÃO D } \\
\text { IDA E SEU EST } \\
\text { A CLASSIFICAC } \\
\text { CRITÉRIO DET } \\
\text { ÓSTICO }(n=8\end{array}$ & $\begin{array}{l}\text { TES SEGUNDO } \\
\text { INSUFICIÊNCIA } \\
\text { DIAMENTO } \\
\text { CO AKIN EM } \\
\text { RMINANTE DO } \\
\text { 5) }\end{array}$ \\
\hline AKIN & n (\%) & $\begin{array}{l}\text { Redução } \\
\text { do débito } \\
\text { urinário }\end{array}$ & $\begin{array}{c}\text { Elevação da } \\
\text { creatinina }\end{array}$ \\
\hline Sem IRA ${ }^{\dagger}$ & $20(23,5)$ & - & - \\
\hline Estágio 1 & $4(4,7)$ & 2 & 2 \\
\hline Estágio 2 & $28(32,9)$ & 28 & 0 \\
\hline Estágio 3 & $33(38,8)$ & 32 & 1 \\
\hline
\end{tabular}

AKIN: Acute Kidney Injury Network; ${ }^{\dagger}$ IRA: Insuficiência Renal Aguda. 
Tabela 5 CARACTERÍSTICAS DO GRUPO DE ACORDO COM A PRESENÇA OU AUSÊNCIA DE INSUFICIÊNCIA RENAL AGUDA

\begin{tabular}{|c|c|c|c|c|}
\hline Características & $\begin{array}{c}\text { IRA ausente } \\
\mathrm{n}(\%)\end{array}$ & $\begin{array}{c}\text { IRA presente } \\
\text { n (\%) }\end{array}$ & Total & Valor $\mathrm{p}^{\dagger}$ \\
\hline Sexo masculino & $11(23,4)$ & $36(76,6)$ & 47 & 0,58 \\
\hline Faixa etária $\geq 60$ anos & $4(11,1)$ & $32(88,8)$ & 36 & 0,02 \\
\hline \multicolumn{5}{|l|}{ Etiologia da $\mathrm{IC}^{\ddagger}$} \\
\hline Valvar & $8(22,2)$ & $28(77,8)$ & 36 & 0,51 \\
\hline Isquêmica & $5(18,5)$ & $22(81,5)$ & 27 & 0,32 \\
\hline Idiopática & $4(40)$ & $6(60)$ & 10 & 0,19 \\
\hline \multicolumn{5}{|l|}{ Comorbidades } \\
\hline$H A S^{\S}$ & $14(26,4)$ & $39(73,6)$ & 53 & 0,29 \\
\hline Anemia & $3(9,1)$ & $30(90,9)$ & 33 & 0,01 \\
\hline IRC" & 0 & $11(100)$ & 11 & 0,04 \\
\hline DPOC & $3(50)$ & $3(50)$ & 6 & 0,13 \\
\hline \multicolumn{5}{|l|}{ Causa da descompensação } \\
\hline Má aderência/ Medicação inadequada & $8(38)$ & $13(62)$ & 21 & 0,07 \\
\hline Disfunção de bioprótese & $1(8,4)$ & $11(91,6)$ & 12 & 0,18 \\
\hline \multicolumn{5}{|l|}{ Fatores de risco } \\
\hline História familiar & $9(33,3)$ & $18(66,7)$ & 27 & 0,12 \\
\hline Tabagismo & $8(22,2)$ & $28(77,8)$ & 36 & 0,51 \\
\hline Etilismo & $2(9)$ & $20(91)$ & 22 & 0,05 \\
\hline \multicolumn{5}{|l|}{ Classe funcional NYHA\# } \\
\hline $\mid$ e || & $5(22,7)$ & $17(77,3)$ & 22 & 0,79 \\
\hline III e IV & $15(24,6)$ & $46(75,4)$ & 61 & 0,71 \\
\hline Hemoglobina admissional* & 12,8 & 11,9 & - & 0,06 \\
\hline Tempo de internação (dias)* & 4,0 & 8,8 & - & $<0,01$ \\
\hline Óbito & $1(5,5)$ & $17(94,5)$ & 18 & 0,04 \\
\hline
\end{tabular}

IRA: Insuficiência Renal Aguda; ${ }^{\dagger}$ Valores considerados significantes com p <0,05; ${ }^{\ddagger}$ IC: Insuficiência Cardíaca; ${ }^{\S} H A S$ : Hipertensão arterial sistêmica; "IRC: Insuficiência Renal Crônica; "DPOC: Doença Pulmonar Obstrutiva crônica; "NYHA: New York Heart Association; *Valores expressos em mediana.

\begin{tabular}{|c|c|c|c|c|}
\hline Tabela 6 & \multicolumn{4}{|c|}{$\begin{array}{l}\text { ANÁLISE ESTATÍSTICA COM MÚLTIPLAS } \\
\text { VARIÁVEIS DE POSSÍVEIS FATORES } \\
\text { PREDITORES DE INSUFICIÊNCIA RENAL } \\
\text { AGUDA EM PACIENTES INTERNADOS POR } \\
\text { INSUFICIEENCIA CARDÍACA DESCOMPENSADA }\end{array}$} \\
\hline \multicolumn{2}{|c|}{ Fatores de risco } & Odds Ratio & IC95\% & Valor $\mathrm{p}^{\dagger}$ \\
\hline \multicolumn{2}{|c|}{ (versus $<60$ anos) } & 0,18 & $0,03-1,10$ & 0,063 \\
\hline \multicolumn{2}{|c|}{ Idiopática } & 0,77 & & 0,84 \\
\hline \multicolumn{2}{|c|}{$\begin{array}{l}\text { Má-aderência/ } \\
\text { medicação inadequada }\end{array}$} & 0,25 & 0,05 & 0,102 \\
\hline \multicolumn{2}{|c|}{$\begin{array}{l}\text { Disfunção de } \\
\text { bioprótese }\end{array}$} & 1,98 & $0,13-29,82$ & 0,620 \\
\hline \multicolumn{2}{|l|}{ DPOC $\ddagger$} & 0,2 & $0,02-$ & \\
\hline \multicolumn{2}{|c|}{ Etilismo } & & & \\
\hline \multicolumn{2}{|c|}{ História familiar Precoce } & 0,46 & $0,09-2,28$ & 0,344 \\
\hline \multicolumn{2}{|c|}{$\begin{array}{l}\text { Hemoglobina } \\
\text { admissional }\end{array}$} & 1,34 & $0,64-2,78$ & 0,438 \\
\hline \multicolumn{2}{|c|}{$\begin{array}{l}\text { Presença de anemia } \\
\text { (versus ausência) }\end{array}$} & 0,11 & $0,01-1,88$ & 0,127 \\
\hline \multicolumn{2}{|c|}{ Tempo de internacão } & 1,72 & $1,08-2,75$ & 0,024 \\
\hline
\end{tabular}

Valores considerados significantes com $p<0,05^{+} ;{ }^{\ddagger}$ DPOC: Doença Pulmonar Obstrutiva Crônica.

\begin{tabular}{|c|c|c|c|}
\hline Tabela 7 & $\begin{array}{l}\text { ANÁLISE ESTATÍS } \\
\text { VARIÁVEIS DE POS } \\
\text { PREDITORES DE N } \\
\text { PACIENTES INTERI } \\
\text { CARDÍACA DESCO }\end{array}$ & $\begin{array}{l}\text { TICA COM MÚL- } \\
\text { SIIVEIS FATORE: } \\
\text { ORTALIDADE EI } \\
\text { JADOS POR INS } \\
\text { MPENSADA }\end{array}$ & $\begin{array}{l}\text { PLAS } \\
\text { FICIÊNCIA }\end{array}$ \\
\hline Fatores de riscc & Odds Ratio & IC95\% & Valor $\mathrm{p}^{+}$ \\
\hline Sexo Masculinc & 1,68 & $0,50-5,59$ & 0,063 \\
\hline $\mathrm{IRC}^{\ddagger}$ & 2,14 & $0,39-11,73$ & 0,844 \\
\hline Tabagismo & 0,56 & $0,17-1,78$ & 0,102 \\
\hline IRA & 0,12 & $0,01-1,05$ & 0,620 \\
\hline
\end{tabular}

${ }^{+}$Nível de significância: $p<0,05$; ${ }^{\prime}$ IRC: Insuficiência renal crônica; s/RA: Insuficiência renal aguda.

\section{Dıscussão}

Neste trabalho em que se analisou a ocorrência de IRA em pacientes internados em UTI por ICD, 76,5\% dos indivíduos desenvolveram a complicação. A redução do débito urinário se relacionou a estágios mais graves e maior mortalidade intra-hospitalar. Havia 12 pacientes com disfunção renal prévia à internação 
e todos eles desenvolveram a complicação. Houve tendência à associação entre IRA e idade acima de 60 anos, e maior tempo de internação hospitalar esteve independentemente associado ao problema. Ter apresentado IRA foi o único fator indepentemente associado à mortalidade intra-hospitalar nesta população.

Vários estudos exploram a deterioração renal em pacientes com IC, ${ }^{14-17}$ porém poucos abordam a ocorrência de IRA no contexto de descompensação da IC. Hata et al., em 2010, por meio da análise retrospectiva de 376 indivíduos admitidos em UTI por ICD, encontraram IRA em $73 \%$ da amostra. ${ }^{12}$ A complicação relacionou-se à elevada mortalidade intra-hospitalar $(10,5 \%$ versus não IRA $1,0 \% ; \mathrm{p}<0,01)$ e tempo de internação prolongado quando comparados ao grupo controle.

Até pouco tempo, o parâmetro mandatório para identificação de lesão renal aguda era a elevação da creatinina sérica. Entretanto, esse biomarcador se altera tardiamente, somente após 24 a 48 horas de injúria, e quando há perda de pelo menos $50 \%$ da função renal. Sua baixa sensibilidade e especificidade implicam diagnóstico e tratamento tardios. ${ }^{16}$ Como quase universalmente utiliza-se esse marcador para avaliação de função renal, um grande percentual de indivíduos que já apresentam a lesão renal aguda subclínica não recebem o diagnóstico e tratamento precoce ou o fazem de modo tardio, com repercussões renais mais graves e aumento do número de óbitos.

Em razão da ausência de outros biomarcadores precoces aplicáveis na prática clínica e da falta de consenso sobre a definição de IRA, Bellomo et al. criaram a classificação RIFLE, que inseriu o débito urinário como medida de avaliação renal. ${ }^{17}$ Partindo desses critérios, Mehta et al. propuseram modificações a fim de antecipar ainda mais o diagnóstico, mediante a nova classificação AKIN. ${ }^{6}$

O débito urinário pode ser medido à beira do leito e sua redução é facilmente percebida clinicamente apesar de sofrer variações conforme o estado volêmico do paciente e uso de diuréticos. Utiliza-se esse marcador em ambos os critérios RIFLE e AKIN, entretanto a acurácia desse parâmetro não é tão abordada em estudos quanto mudanças nos valores séricos de creatinina, por não estar sempre disponível para análise nos prontuários.

IRA ocorreu em estágios AKIN mais avançados na maior parte dos pacientes acometidos, e o critério mais utilizado para a sua detecção foi a redução do débito urinário. A percepção da sua ocorrência somente nos estágios AKIN mais avançados pode se justificar pelo uso frequente de diuréticos no manejo terapêutico de pacientes com IC. ${ }^{18,19}$ Apesar de fazer parte do tratamento de ICD, ${ }^{3}$ diuréticos não propiciam incremento na clínica ou na recuperação após IRA, sendo eficaz apenas como estímulo à diurese e redução de distúrbios hidroeletrolíticos na presença de sobrecarga volêmica. ${ }^{10,19}$ De fato, seu uso pode implicar retardo na percepção da alteração urinária e, consequentemente, do diagnóstico de IRA. O critério débito urinário mostrou ser marcador mais precoce por Magro et al. e Santos et al. em análise de IRA no pós-operatório de cirurgia cardíaca. ${ }^{20,21}$

Outra hipótese é que existam pacientes com doença renal crônica (DRC) não identificada, que por isso apresentaram IRA em estágios avançados. DRC é cada vez mais comum em pacientes portadores de IC e pode interferir na intensidade dos sintomas, mudar o curso clínico e alterar a resposta ao tratamento. ${ }^{22}$ Nesta amostra, todos os indivíduos que tinham disfunção renal prévia evoluíram com IRA. Esses resultados podem ser justificados pela indevida valorização da função renal na consulta de atenção primária e nos serviços especializados. Essa avaliação faz parte da rotina laboratorial do paciente com IC, ${ }^{23}$ que pode não estar sendo feita de forma adequada. A disfunção renal é considerada fator independente para prognóstico ruim em pacientes hospitalizados por ICD, ${ }^{24,25}$ além de preditor da ocorrência de IRA em UTI. ${ }^{26,27}$ Em trabalho prévio realizado na mesma instituição com pacientes internados por síndrome coronariana aguda, a creatinina sérica só foi dosada na admissão em apenas $50,3 \%$ da amostra. ${ }^{28}$ Esse fato se deve à falta de atenção, por parte dos cardiologistas, à avaliação da função renal, mesmo sabendo que essa população possui alto risco para DRC.

Nesta casuística, a idade superior a 60 anos tendeu a ser fator preditor independente de IRA, resultado encontrado também por Hata et al., Lopes et al. e Abosaif et al...,9,12 Alterações na musculatura vascular em decorrência do processo de envelhecimento e outras condições, como diabetes mellitus, aterosclerose e hipertensão crônica, justificam a queda da efetividade com o tempo do mecanismo de autorregulação renal, fato que predispõe ao aparecimento de IRA em quedas mínimas de pressão arterial. ${ }^{11}$

A presença de anemia em IC associa-se a disfunção renal, diabetes mellitus, idade avançada e classe NYHA mais alta. ${ }^{29-31}$ Sales et al. identificaram anemia como preditor independente de mortalidade em ICD. ${ }^{30}$ Em nosso trabalho, 38,8\% dos pacientes apresentaram anemia e houve associação com a presença de IRA. Contudo, sua ligação com eventos adversos não está clara, pois a anemia pode ser apenas um 
marcador de maior gravidade da IC ou mais uma comorbidade que piora o prognóstico.

Tempo de internação prolongada foi fator independente para ocorrência de IRA, entretanto não podemos diferenciar se a permanência hospitalar prolongada em si predispôs a ocorrência de IRA ou se os pacientes ficaram mais tempo internados na UTI porque desenvolveram a complicação. Indivíduos acometidos por IRA apresentaram pior desfecho clínico, e a complicação esteve relacionada independentemente ao risco de óbito, assim como descrito em outros trabalhos. ${ }^{7-9}$ Recentemente, Garzotto et al. evidenciaram maior tempo de internação em pacientes críticos que desenvolveram IRA (média 7 dias versus não IRA 3 dias; $p<0,01$ ) e sua relação positiva com estágios mais avançados e maior mortalidade. ${ }^{8}$ Este resultado foi comprovado também por Bagshaw et al. em grande estudo de coorte prospectivo que analisou pacientes admitidos em UTI na Austrália. ${ }^{32}$

Dentre as limitações do nosso trabalho, citamos a ausência de informações sobre as medicações utilizadas pelos pacientes e o número relativamente reduzido da amostra analisada, entretanto deve-se considerar que se tratou de estudo prospectivo realizado em uma única instituição. Pesquisas com maior abrangência devem ser conduzidas a fim de investigar a habilidade do critério AKIN em diagnosticar IRA precocemente na ICD. Pela facilidade de aplicação e baixo custo, este critério mostra-se como ferramenta promissora no manejo do paciente com ICD.

Em síntese, IRA avaliada pelo critério AKIN se fez complicação presente na maioria dos pacientes críticos hospitalizados por ICD e associou-se a maior tempo de internação e número de óbitos. O débito urinário foi o parâmetro mais utilizado na sua detecção, que ocorreu em estágios mais avançados. Todos os portadores de IRC desenvolveram a complicação. Tempo de hospitalização em UTI e idade acima de 60 anos estiveram associados ao problema. IRA foi fator preditor independente de mortalidade nos pacientes internados por ICD em UTI.

\section{Conclusão}

IRA é entidade frequentemente associada à ICD, ocorre nos estágios mais avançados, notadamente em indivíduos renais crônicos, idosos e com maior tempo de internação, e relaciona-se a maior mortalidade. A utilização rotineira de critérios diagnósticos simples, como a classificação AKIN, deve ser encorajada para que se tenha uma melhor prevenção e um diagnóstico mais precoce de IRA nessa população específica.

\section{REFERÊNCIAS}

1. Spineti PPM, Tedeschi B, Sales ALF, et al. Incidência e Preditores de Síndrome Cardiorrenal Aguda durante Tratamento de Insuficiência Cardíaca Descompensada: análise de 332 hospitalizações consecutivas. Rev SOCERJ 2009;22:93-8.

2. DATASUS. Departamento de Informática do SUS. Ministério da Saúde. [cited 2011 Sep 14]. Available from: www.datasus.gov.br.

3. Bocchi EA, Vilas-Boas F, Perrone S, et al. I Diretriz Latino-Americana para avaliação e conduta na insuficiência cardíaca descompensada. Arqu Bras Cardiol 2005;85:1-48.

4. Abosaif NY, Tolba YA, Heap M, Russell J, Nahas ME. The outcome of acute renal failure in the intensive care unit according to RIFLE: model application, sensitivity, and predictability. Am J Kidney Dis 2005;46:1038-48.

5. Bellomo R, Ronco C, Kellum JA, Mehta RL, Palevsky $P$. Acute renal failure - definition, outcome measures, animal models, fluid therapy and information technology needs: the Second International Consensus Conference of the Acute Dialysis Quality Initiative (ADQI) Group. Crit Care 2004;8:R204-12.

6. Mehta RL, Kellum JA, Shah SV, et al. Acute Kidney Injury Network: report of an initiative to improve outcomes in acute kidney injury. Crit Care 2007;11:R31.

7. Bagshaw SM, George C, Bellomo R. A comparison of the RIFLE and AKIN criteria for acute kidney injury in critically ill patients. Nephrol Dial Transplant 2008;23:1569-74.

8. Garzotto F, Piccinni P, Cruz D, et al. RIFLE-based data collection/management system applied to a prospective cohort multicenter Italian study on the epidemiology of acute kidney injury in the intensive care unit. Blood Purif 2011;31:159-71.

9. Lopes JA, Fernandes P, Jorge S, et al. Acute kidney injury in intensive care unit patients: a comparison between the RIFLE and the Acute Kidney Injury Network classifications. Crit Care 2008;12:R110.

10. Ronco C, Maisel A. Volume overload and cardiorenal syndromes. Congest Heart Fail 2010;16:Si-iv.

11. Macedo E, Mehta R. Prerenal azotemia in congestive heart failure. Contrib Nephrol 2010;164:79-87.

12. Hata N, Yokoyama S, Shinada T, et al. Acute kidney injury and outcomes in acute decompensated heart failure: evaluation of the RIFLE criteria in an acutely ill heart failure population. Eur J Heart Fail 2010;12:32-7.

13. Carlson KJ, Lee DC-S, Goroll AH, Leahy M, Johnson RA. An analysis of physicians' reasons for prescribing long-term digitalis therapy in outpatients. J Chronic Dis 1985;38:733-9.

14. Aronson D, Burguer AJ. The relationship between transient and persistent worsening renal function and mortality in patients with acute decompensated heart failure. J Card Fail 2010;16:541-7.

15. Bobadilla JF, López-Sendón J. Enfermedad renal: implicaciones terapéuticas en insuficiencia cardíaca y cardiopatía isquémica. Med Clin (Barc) 2009;132:48-54.

16. Dries DL, Exner DV, Domanski MJ, Greenberg B, Stevenson LW. The prognostic implications of renal insufficiency in asymptomatic and symptomatic 
patients with left ventricular systolic dysfunction. J Am Coll Cardiol 2000;35:681-9.

17. Goldberg RJ, Ismailov RM, Patlolla V, Lessard D, Spencer FA. Therapies for acute heart failure in patients with reduced kidney function: a community-based perspective. Am J Kidney Dis 2008;51:594-602.

18. Magro MCS, Vattimo MFF. Avaliação da Função Renal: Creatinina e outros Biomarcadores. Revista Brasileira de Terapia Intensiva 2007;19:182-5.

19. Prowle JR, Echeverri JE, Ligabo EV, Ronco C, Bellomo R. Fluid Balance and acute kidney injury. Nat Rev Nephrol 2010;6:107-15.

20. Magro MCS, Franco ES, Guimarães D, Kajimoto D, Gonçalves MAB, Vattimo MFF. Avaliação da função renal em pacientes no pós-operatório de cirurgia cardíaca: a classificação AKIN prediz disfunção renal aguda? Revista Brasileira de Terapia Intensiva 2009;21:25-31.

21. Santos IM. Insuficiência renal aguda no pós-operatório de cirurgia cardíaca determinada através da aplicação do critério RIFLE [monografia]. Aracaju: Universidade Federal de Sergipe; 2008.

22. Tello BS, Sales ALF, Barcellos E, et al. Anemia e Disfunção Renal na Insuficiência Cardíaca. Rev SOCERJ 2007;20:434-42.

23. Bocchi EA, Marcondes-Braga FGM, Ayub-Ferreira SMA, et al. III Diretriz Brasileira de Insuficiência Cardíaca Crônica. Arq Bras Cardiol 2009;92:7-71.

24. Smith GL, Lichtman JH, Bracken MB, et al. Renal impairment and outcomes in heart failure: systematic review and meta-analysis. J Am Coll Cardiol 2006;47:1987-96.
25. Silverberg DS, Wexler D, Blum M, et al. The use of subcutaneous erythropoietin and intravenous iron for the treatment of the anemia of severe, resistant congestive heart failure improves cardiac and renal function and functional cardiac class, and markedly reduces hospitalizations. J Am Coll Cardiol 2000;35:1737-44.

26. Himmelfarb J, Ikizler TA. Acute kidney injury: changing lexicography, definitions, and epidemiology. Kidney Int 2007;71:971-6.

27. Xue JL, Daniels F, Star RA, et al. Incidence and mortality of acute renal failure in Medicare beneficiaries, 1992 to 2001. J Am Soc Nephrol 2006;17:1135-42.

28. Cruz EV. Disfunção Renal em Pacientes com Síndrome Coronariana Aguda [monografia]. Aracaju: Universidade Federal de Sergipe; 2007.

29. Reis FJFB, Fernandes AMS, Bitencourt AGV, et al. Prevalência de anemia e insuficiência renal em portadores de insuficiência cardíaca não-hospitalizados. Arq Bras Cardiol 2009;93:268-74.

30. Sales ALF, Villacorta H, Reis L, Mesquita ET. Anemia como fator prognóstico em uma população hospitalizada por insuficiência cardíaca descompensada. Arq Bras Cardiol 2005;84:237-40.

31. Tang YD, Katz SD. Anemia in chronic heart failure: prevalence, etiology, clinical correlates, and treatment options. Circulation 2006;113:2454-61.

32. Bagshaw SM, George C, Dinu I, Bellomo R. A multicentre evaluation of the RIFLE criteria for early acute kidney injury in critically ill patients. Nephrol Dial Transplant 2008;23:1203-10. 